\title{
Desmoplastic Melanoma Masquerading as Neurofibroma
}

\author{
Evan Stokar, MD; Carlos Rodriguez, MD; Jerry Feldman, MD
}

\section{PRACTICE POINTS}

- Desmoplastic melanoma remains a diagnostic challenge both clinically and histologically.

- New or changing lesions on sun-exposed sites of elderly patients with fair skin types should have a low threshold for biopsy.

- Consensus between more than one dermatopathologist is sometimes required to make the diagnosis histologically.
Desmoplastic melanoma (DMM) is a rare variant of melanoma that presents major diagnostic challenges. It can mimic both benign or other malignant tumors and may be easily confused by clinicians and pathologists. We report a patient who presented with a pink-colored papule on the left lateral neck. Histopathology revealed a nodular spindle cell tumor in the dermis resembling a neurofibroma at low power. However, higher-power evaluation and the use of immunohistochemical staining confirmed the diagnosis of DMM.

We present this case to highlight the ongoing challenges of diagnosing DMM both clinically and histologically and to review the salient features of this often benign-appearing tumor. Advancing age, male gender, and head and neck location are associated with an increased risk of DMM-specific death. It is important that new or changing lesions in the correct cohort and location are biopsied promptly.

Cutis. 2021;107:258-260.

$\square$ esmoplastic melanoma (DMM) is a rare variant of melanoma that presents major challenges to both clinicians and pathologists. ${ }^{1}$ Clinically, the lesions may appear as subtle bland papules, nodules, or plaques. They can be easily mistaken for benign growths, leading to a delayed diagnosis. Consequently, most DMMs at the time of diagnosis tend to be thick, with a mean Breslow depth ranging from 2.0 to $6.5 \mathrm{~mm}$. $^{2}$ Histopathologic evaluation has its difficulties. At scanning magnification, these tumors may show low cellularity, mimicking a benign proliferation. It is well recognized that S-100 and other tumor markers lack specificity for DMM, which can be positive in a range of neural tumors and other cell types. ${ }^{2}$ In some amelanotic tumors, DMM becomes virtually indistinguishable from benign peripheral sheath tumors such as neurofribroma. ${ }^{3}$

Desmoplastic melanoma is exceedingly uncommon in the United States, with an estimated annual incidence rate of 2.0 cases per million. ${ }^{2}$ Typical locations of presentation include sun-exposed skin, with the head and neck regions representing more than half of reported cases. ${ }^{2}$ Desmoplastic melanoma largely is a disease of fairskinned patients, with $95.5 \%$ of cases in the United States occurring in white non-Hispanic individuals. Advancing age, male gender, and head and neck location are associated with an increased risk for DMM-specific death. ${ }^{2}$ It is important that new or changing lesions in the correct cohort and location are biopsied promptly. We present this case to highlight the ongoing challenges of diagnosing DMM both clinically and histologically and to review the salient features of this often benign-appearing tumor.

\section{Case Report}

A 51-year-old White man with a history of prostate cancer, a personal and family history of melanoma, and benign neurofibromas presented with a 6-mm, pink, well-demarcated, soft papule on the left lateral neck (Figure 1). The lesion had been stable for many years but began growing more rapidly 1 to 2 years prior to

Drs. Stokar and Feldman are from the Division of Dermatology, John H. Stroger, Jr. Hospital of Cook County, Chicago, Illinois. Dr. Rodriguez is from Arrowhead Dermatology, Phoenix, Arizona.

The authors report no conflict of interest.

Correspondence: Evan Stokar, MD, 1900 W Polk St, Room 519, Chicago, IL 60612 (evan.stokar@gmail.com).

doi:10.12788/cutis.0241 
presentation. The lesion was asymptomatic, and he denied changes in color or texture. There also was no bleeding or ulceration. A review of systems was unremarkable. A shave biopsy of the lesion revealed a nodular spindle cell tumor in the dermis resembling a neurofibroma on low power (Figure 2). However, overlying the tumor was a confluent proliferation positive for MART-1 and S-100, which was consistent with a diagnosis of melanoma in situ (Figure 3). Higher-power evaluation of the dermal proliferation showed both bland and hyperchromatic spindled and epithelioid cells (Figure 4), with rare mitotic figures highlighted by $\mathrm{PHH}$, an uncommon finding in neurofibromas (Figure 5). The dermal spindle cells were positive for S-100 and p75 and negative for Melan-A. Epithelial membrane antigen highlighted a faint sheath surrounding the dermal component. Ki-67 revealed a mildly increased proliferative index in the dermal component. The diagnosis of DMM was made after outside dermatopathology consultation was in agreement. However, the possibility of a melanoma in situ growing in association with an underlying neurofibroma remained a diagnostic consideration histologically. The lesion was widely excised.

\section{Comment}

Differential for DMM-Early DMMs may not show sufficient cytologic atypia to permit obvious distinction from neurofibromas, which becomes problematic when encountering a spindle cell proliferation within severely sun-damaged skin, or even more so when an intraepidermal population of melanocytes is situated above a dermal population of slender, spindled, S-100-positive cells, as seen in our patient. ${ }^{4}$ For these challenging scenarios, Yeh and McCalmont ${ }^{4}$ have proposed evaluating for a CD34 "fingerprint" pattern. This pattern typically is widespread in neurofibroma but absent or limited in DMM, and it is a useful adjunct in the differential diagnosis when conventional immunohistochemistry has little contribution.

There are several case reports in the literature of DMM mimicking other benign or malignant proliferations. In 2012, Jou et $\mathrm{al}^{5}$ described a case of a 62 -yearold White man who presented with an oral nodule consistent with fibrous inflammatory hyperplasia clinically. Incisional biopsy later confirmed the diagnosis of amelanotic DMM. ${ }^{5}$ Similar case reports have been described in which the diagnosis of DMM was later found to resemble a sarcoma and malignant peripheral nerve sheath tumor. ${ }^{6,7}$

Diagnosis of DMM-The prototypical DMM is an asymmetrical and deeply infiltrative spindle cell lesion in severely sun-damaged skin. By definition, the individual melanocytes are separated by connective tissue components, giving the tumor a paucicellular appearance. ${ }^{1}$ Although the low cellularity can give a deceptively bland scanning aspect, on high-power examination there usually are identifiable atypical spindled cells with enlarged,

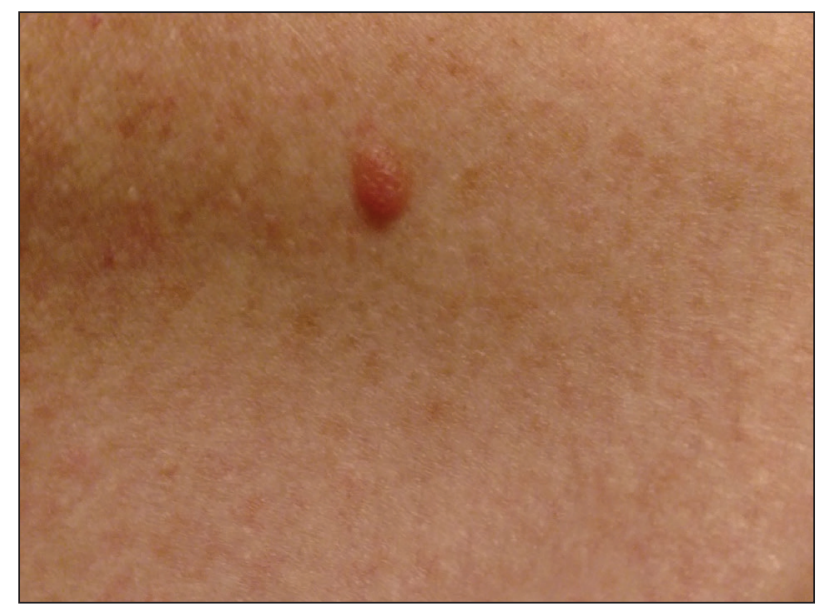

FIGURE 1. A 6-mm, pink, well-demarcated, soft papule on the left lateral neck.

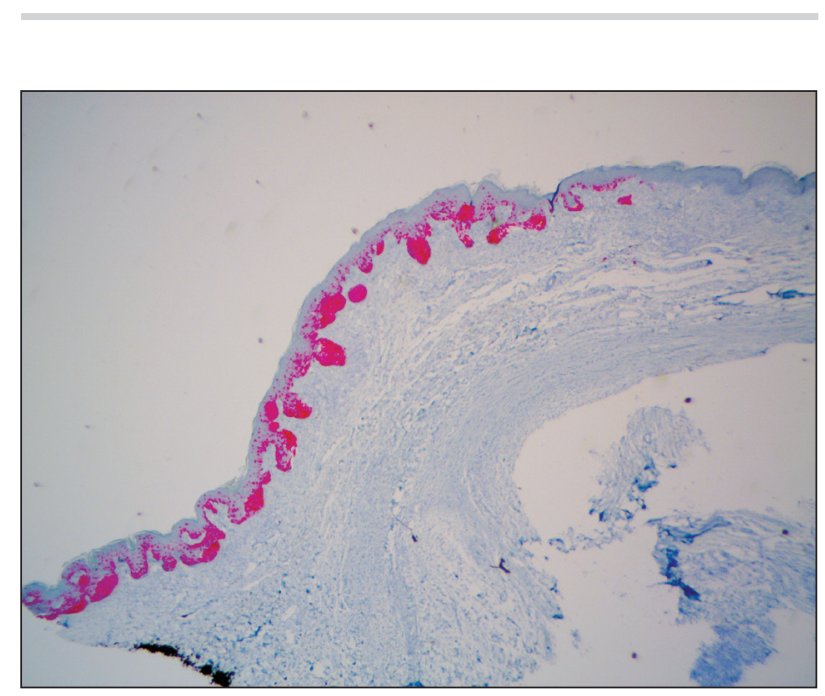

FIGURE 2. Low-power histologic evaluation revealed a nodular spindle cell tumor in the dermis $(H \& E$, original magnification $\times 4)$.

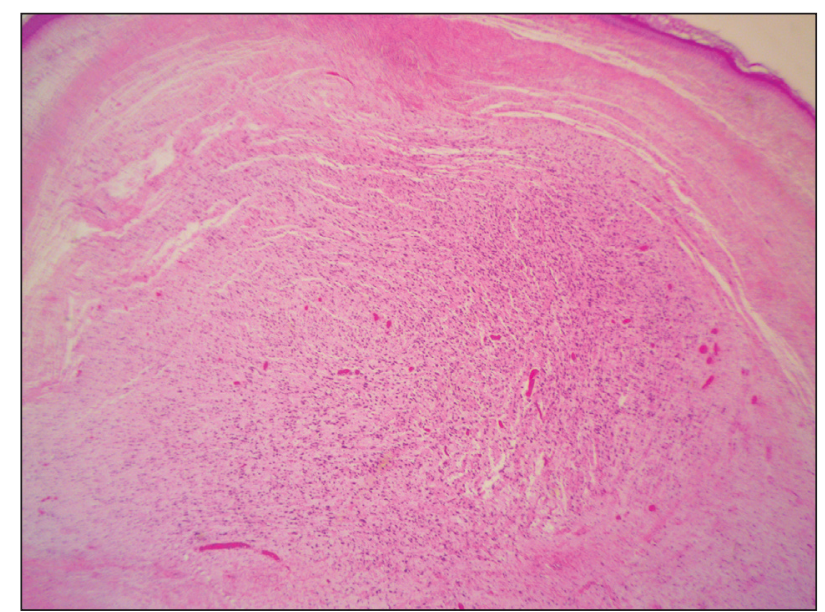

FIGURE 3. MART-1-positive proliferation overlying the dermal tumor (original magnification $\times 10$ ). 


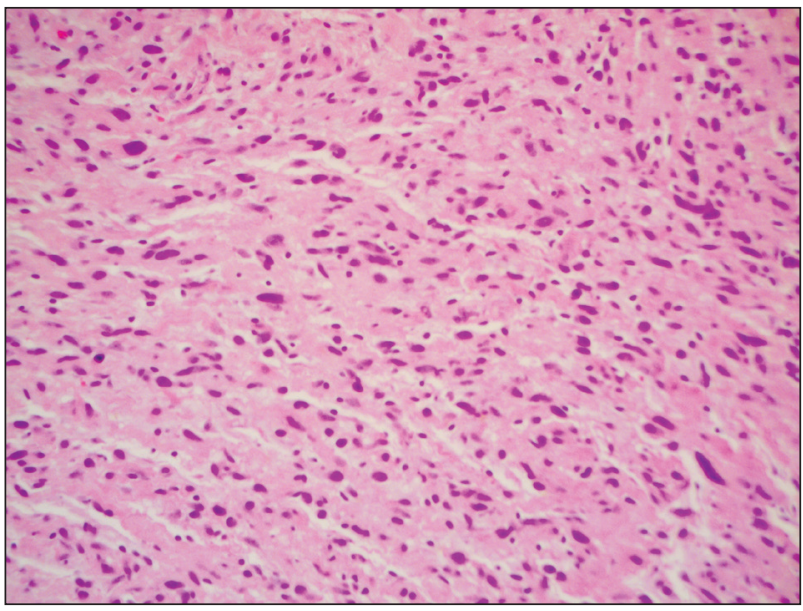

FIGURE 4. Many hyperchromatic spindled and epithelioid cells (H\&E, original magnification $\times 20$ ).

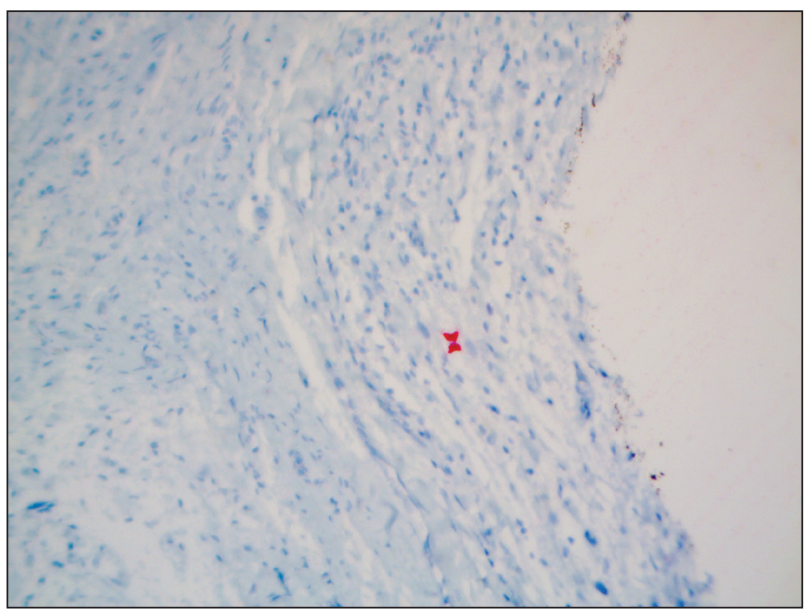

FIGURE 5. $\mathrm{PHH} 3$ immunostain highlighted a rare mitotic figure within the dermal proliferation (original magnification $\times 20$ ).

elongated, and hyperchromatic nuclei. S-100 typically is diffusely positive in DMM, though occasional cases show more limited staining. ${ }^{8}$ Other commonly used and more specific markers of melanocytic differentiation, including HMB45 and Melan-A, typically are negative in the paucicellular spindle cell components. ${ }^{9}$ Desmoplastic melanoma can be further categorized by the degree of fibrosis within a particular tumor. If fibrosis is prominent throughout the entire tumor, it is named pure DMM. On the other hand, fibrosis may only represent a portion of an otherwise nondesmoplastic melanoma, which is known as combined DMM. ${ }^{10}$

\section{Conclusion}

We present this case to highlight the ongoing challenges of diagnosing DMM both clinically and histologically. Although a bland-appearing lesion, key clinical features prompting a biopsy in our patient included recent growth of the lesion, a personal history of melanoma, the patient's fair skin type, a history of heavy sun exposure, and the location of the lesion. According to Busam, ${ }^{11}$ an associated melanoma in situ component is identified in $80 \%$ to $85 \%$ of DMM cases. Detection of a melanoma in situ component associated with a malignant spindle cell tumor can help establish the diagnosis of DMM. In the absence of melanoma in situ, a strong diffuse immunoreactivity for S-100 and lack of epithelial markers support the diagnosis. ${ }^{11}$ After review of the literature, our case likely represents DMM as opposed to a melanoma in situ developing within a neurofibroma.

\section{REFERENCES}

1. Wood BA. Desmoplastic melanoma: recent advances and persisting challenges. Pathology. 2013;45:453-463.

2. Chen LL, Jaimes N, Barker CA, et al. Desmoplastic melanoma: a review. J Am Acad Dermatol. 2013;68:825-833.

3. Machado I, Llombart B, Cruz J, et al. Desmoplastic melanoma may mimic a cutaneous peripheral nerve sheath tumor: report of 3 challenging cases. J Cutan Pathol. 2017;4:632-638.

4. Yeh I, McCalmont, TH. Distinguishing neurofibroma from desmoplastic melanoma: the value of the CD34 fingerprint. J Cutan Pathol. 2011;38:625-630

5. Jou A, Miranda FV, Oliveira MG, et al. Oral desmoplastic melanoma mimicking inflammatory hyperplasia. Gerodontology. 2012;29:E1163-E1167.

6. Ishikura H, Kojo T, Ichimura H, et al. Desmoplastic malignant melanoma of the uterine cervix: a rare primary malignancy in the uterus mimicking a sarcoma. Histopathology. 1998;33:93-94.

7. Barnett SL, Wells MJ, Mickey B, et al. Perineural extension of cutaneous desmoplastic melanoma mimicking an intracranial malignant peripheral nerve sheath tumor. case report. J Neurosurg. 2011;115:273-277.

8. Jain S, Allen PW. Desmoplastic malignant melanoma and its variants. a study of 45 cases. Am J Surg Pathol. 1989;13:358-373.

9. Skelton HG, Maceira J, Smith KJ, et al. HMB45 negative spindle cell malignant melanoma. Am J Dermatopathol. 1997; 19:580-584.

10. George E, McClain SE, Slingluff CL, et al. Subclassification of desmoplastic melanoma: pure and mixed variants have significantly different capacities for lymph node metastasis. J Cutan Pathol. 2009;36:425-432.

11. Busam KJ. Desmoplastic melanoma. Clin Lab Med. 2011;31:321-330. 